\title{
Investigation on industrial dataspace for advanced machining workshops: enabling machining operations control with domain knowledge and application case studies
}

\author{
Pulin $\mathrm{Li}^{1} \cdot$ Kai Cheng ${ }^{2} \cdot$ Pingyu Jiang ${ }^{1}$ (D) Kanet Katchasuwanmanee ${ }^{2}$
}

Received: 14 November 2019 / Accepted: 13 August 2020

(c) Springer Science+Business Media, LLC, part of Springer Nature 2020

\begin{abstract}
The machining processes on the advanced machining workshop floor are becoming more sophisticated with the interdependent intrinsic processes, generation of ever-increasing in-process data and machining domain knowledge. To manage and utilize those above effectively, an industrial dataspace for machining workshop (IDMW) is presented with a three-layer framework. The IDMW architecture is Schema Centralized-Data Distributed, which relies on Process-Workpiece-Centric knowledge schema description and data storage in decentralized data silos. Subsequently, the pre-processing method for the data silos driven by RFID event graphical deduction model is elaborated to associate decentralized data with knowledge schema. Furthermore, through two industrial case studies, it is found that IDMW is effective in managing heterogeneous data, interconnecting the resource entities, handling domain knowledge, and thereby enabling machining operations control on the machining workshop floor particularly.
\end{abstract}

Keywords Industrial dataspace Machining knowledge $\cdot$ Machining operations control $\cdot$ Knowledge representation · Knowledge graph

\section{List of symbols}
$\mathrm{A} \Theta \mathrm{B}$
$c t a b c$
$f t a b c$
mm_abc
mstq_abc
mps_abc
$m t a b c$
$m t s \_a b c$
$m p \_a b c$
$q f_{-} a b c$
$r X X$
$s X X$
URI of cutting tool $a b c$
URI of feature $a b c$
URI of machining methods $a b c$
URI of quality measure tool $a b c$
URI of machining process status $a b c$
URI of machining tool $a b c$
URI of measure tool/sensor $a b c$
URI of machining process $a b c$
URI of quality feature $a b c$
The $X X$ th response to $s X X$
The $X X$ th operation sequence

Pingyu Jiang

pjiang@mail.xjtu.edu.cn

1 State Key Laboratory for Manufacturing Systems Engineering, Xi'an Jiaotong University, Xi'an, Shaanxi, China

2 College of Engineering, Design and Physical Science, Brunel University London, Uxbridge UB8 3PH, UK
$t_{P}^{s}$

$t_{P}^{s i}$

$s_{P}^{s} \quad$ Arriving timeline from the last process to the current process $p$

Starting timeline of loading workpiece to machine tool table in process $p$

$t_{P}^{e i} \quad$ Ending timeline of loading workpiece in machine tool table in process $p$

$t_{P}^{s p} \quad$ Starting timeline of processing the workpiece in process $p$

$t_{P}^{e p} \quad$ Ending timeline to processing workpiece in process $p$

$t_{P}^{s o} \quad$ Starting Timeline of putting workpiece off machine tool table in process $p$

$t_{P}^{e o} \quad$ Ending timeline of putting workpiece off machine tool table in process $p$

$t_{P}^{e} \quad$ Leaving timeline from the current process $p$

$T_{P-1}^{p} \quad$ Transportation time from process $p-1$ to process $p$

$T_{P}^{x} \quad$ Processing time of $x$ in process $p$

$w k a b c \quad$ URI of worker $a b c$

$w p \_a b c \quad$ URI of workpiece $a b c$ 


\section{Abbreviations}

$\begin{array}{ll}\text { APP } & \text { Applications } \\ \text { CNC } & \text { Computer numerical control } \\ \text { CPS } & \text { Cyber-physical-system } \\ \text { ERP } & \text { Enterprise resource planning } \\ \text { IDMW } & \text { Industrial dataspace for machining workshop } \\ \text { MEPN } & \text { Machining error propagation network } \\ \text { MES } & \text { Manufacturing execution systems } \\ \text { MOC } & \text { Machining operations control } \\ \text { NGIT } & \text { New generation of information technology } \\ \text { OBDA } & \text { Ontology-based data access } \\ \text { OWL } & \text { Web ontology language } \\ \text { RFID } & \text { Radio frequency identification } \\ \text { SQL } & \text { Structured query language } \\ \text { URI } & \text { Uniform resource identifier }\end{array}$

\section{Introduction}

Several strategies about advanced manufacturing have been initiated worldwide recently, such as Industry 4.0 in Germany (Zhong et al. 2017a, b), Industrial Internet in the USA (Xu and Duan 2019), the Made in China 2025 (Zhou et al. 2018), and High-Value Manufacturing in the UK (UK 2012). The implementation of those initiatives encourages the New Generation of Information Technology (NGIT), representing by cyber-physical-system (CPS) (Lu and Xu 2018), Big data (Wang et al. 2016), and Knowledge Graph (Song et al. 2018), to be used in manufacturing (Ye and Wang 2013). With the NGITs' help, collecting and processing all kinds of machining in-process data in real-time have become practicable. NGITs also show massive potential in enabling intelligent machining operations control (MOC) and thereby improving the quality and efficiency of ever-increasing complex machining processes on the advanced machining workshop floor.

Data management, especially in the context of big data (Flyverbom et al. 2017), plays a more and more crucial role in intelligent MOC. In an advanced machining workshop, the decision-making instructions should not rely on probabilistic data (Ji et al. 2019). It would be unacceptable if only using probabilistic uncertainty instructions to guide a real-world machining process. Furthermore, the machining in-process data have the feature of massive and varying, but the machining relations of resource entities seem relatively regular (Qi and Tao 2018). Besides, the machining is a specialized discipline, containing lots of expertise (domain knowledge). How to discover, represent, store and reuse this kind of domain knowledge and thereby to handle the in-process knowledge need to be taken into consideration when applying data management in advanced machining workshops.
However, there are no specific ways of linking the machining in-process data with domain knowledge. For the connection between the raw machining data and the final performance of quality and efficiency, the industrial dataspace for the machining workshop (IDMW) is introduced with the help of machining domain knowledge modeling on the machining workshop floor. The term dataspace is defined as the handling of knowledge schema and complex data in data management. Accordingly, the IDMW is designed for tackling the MOC issue through managing the heterogeneous data, interconnecting the resource entities, and handling the machining domain knowledge. With it, data can be sensed and stored while knowledge can be represented and reused effectively.

This paper will focus on the IDMW and its enabling technologies. The IDMW not only concerns the NGITs but also takes the manufacturing logics and machining mechanisms into consideration. The rest of the paper will be arranged as follows. "Problems to be solved and critical review of related work" section summarises some challenging issues to be solved and critically reviews the related works. "Construction of the IDMW" section describes how to construct the IDMW from the aspects of the framework, relation description, and distributed data storage. "Pre-processing of the data silos during the runtime of IDMW" section elaborates the Pre-processing of the data silos during the runtime of IDMW. Furthermore, two industrial cases in different machining domains are described in "Industrial case studies" section to illustrate the application of the IDMW. Finally, further discussions and conclusions are given. The IDMW explains how to associate machining in-process data with domain knowledge. It is good at managing heterogeneous data, interconnecting the resource entities, and handling domain knowledge on the advanced machining workshop floor particularly.

\section{Problems to be solved and critical review of related work}

\section{Problems to be solved}

The development of NGIT can drive the machining process to be more intelligent and controllable. However, there are some challenging issues to be solved when carrying out datadriven advanced machining, which illustrated in Fig. 1.

(1) Management of different varieties of in-process data in a machining workshop.

Data are continually generated all the time, whether machining or not. Each resource entity in a machining workshop can generate data (Leng et al. 2019), which sources and 


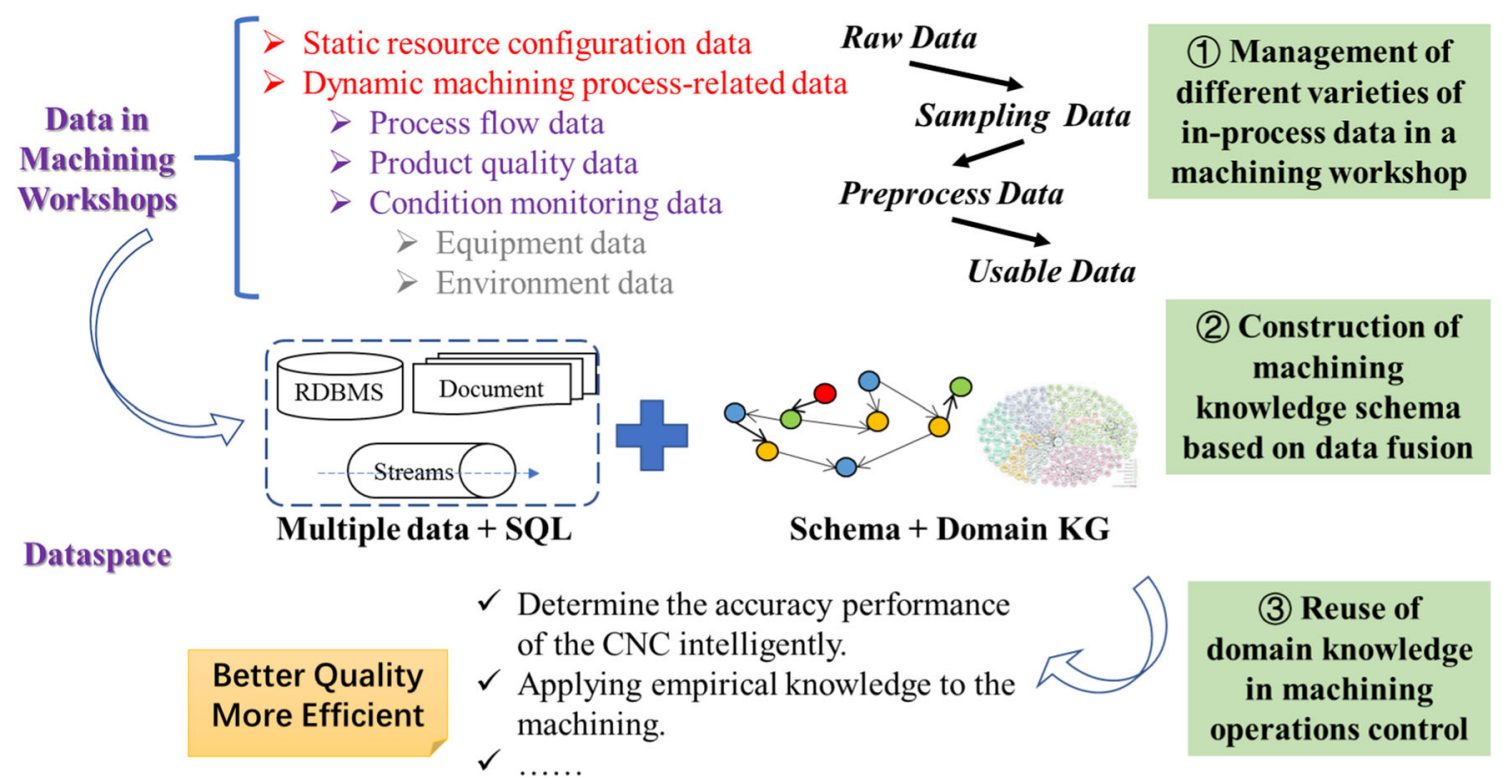

Fig. 1 Three challenging issues to be solved when carrying out data-driven advanced machining

formats are generally diverse. Briefly, there are two main categories, which are static resource configuration data and dynamic machining process-related data. The dynamic ones can also be sub-divided into process flow data, product quality data, and condition monitoring data. How to methodically sense, collect, store and manage these kinds of heterogeneous data is the first challenging issue to be solved.

(2) Construction of machining knowledge schema based on data fusion.

The heterogeneous data are hardly reflecting the machining process before being fused. Data will be stored in databases, documents, and files located in different places, so it is needed to use a knowledge schema to interconnect the segregated data (Li et al. 2019). Machining knowledge schema can represent the relations of resource entities within the workshop. Thus, how to construct the proper machining knowledge schema that can fuse data is the second challenging issue.

(3) Reuse of domain knowledge in machining operations control.

There is a lot of domain knowledge about the machining processes, e.g., the influence of tooling, fixtures, materials, machining parameters on precision machining performance. All the domain knowledge can be expressed as the relations of resource entities in the knowledge schema. Thus, the third challenging issue is how to reuse the domain knowledge in machining operations control.

\section{Critical review of related work}

Dataspace means the space of data originally, which is an abstraction in data management that aims to overcome some of the problems encountered in the data integration system in the field of informology (Belhajjame et al. 2013). Dataspaces can be viewed as the next stage of data integration, enabling data intelligence (Curry 2020). Moreover, the industrial dataspace has not been proposed until recent years while this section will elaborate on some relevant works from the following three aspects.

\section{Machining in-process data collection, storage, and management}

In-process data are continuously generating during machining, and the NGITs make them possible to be sensed and collected. These in-process data are with the features of massive, multi-source, high-dimensional, and heterogeneous, due to the complex interactions between production orders and machining processes (Zhong et al. 2017a, b). Some researchers have focused on real-time data collecting, mining, analyzing, storing, and managing during machining processes (Babiceanu and Seker 2016). The CPS is configured for collecting data timely, relying on different kinds of sensing devices, such as radio frequency identification (RFID), sensors, and laser scanners. (Liu et al. 2017). For instance, Lee (Lee et al. 2015) advanced a unified 5-level CPS architecture as a guideline for manufacturing. And a CPS-driven architecture based on CNC machining data was proposed to achieve the intelligent MOC (Zhang et al. 2016). Besides, information systems, represented by manufacturing 
execution systems (MES) and enterprise resource planning (ERP), play the role of managing the complex data, tracking and documenting the transformation of raw materials to finished goods (Qiu and Zhou 2004). Their affiliated databases accept responsibility for saving different kinds of data (Tao et al. 2018). MES and ERP have received considerable attention that several software vendors such as SAP, Oracle, Siemens have released mature products (Parthiban and Nataraj 2019). Moreover, some researchers have proposed novel data solutions based on big data tools (Cui et al. 2020). And some scholars also have put forward distributed architectures for data management within the machining workshop (Kovalenko et al. 2019; Mezgebe et al. 2019; Novas et al. 2012). For example, Novas has succeeded in using a multiagent system to develop a collaborative system (Novas et al. 2012).

In summary, with the NGITs' help, machining in-process data will be sensed, collected, stored, and managed. However, these kinds of in-process data are distributed, heterologous, and heterogeneous, which is difficult to be handled in existing data systems. Besides, the scale of the in-process data is also too large to store in the traditional database. In addition, there is little research on discovering implicit relations among different types of data in different locations.

\section{Machining domain knowledge representation}

Domain knowledge can make machining operations more intelligent. Many expert systems have succeeded in describing knowledge with specific formats, storing them in the structural database, and sharing them with others for reusing (Gao and Nee 2017; Evans et al. 2017; Zammit et al. 2017; He and Jiang 2019). Besides, the knowledge graph, as one of the state-of-the-art technologies, is becoming a popular way to express large-scale knowledge (Nickel et al. 2016). Some domain knowledge graphs were also studied in the fields of the additive manufacturing (Wang et al. 2018), product design (Chhim et al. 2019), service-oriented business interactions ( $\mathrm{Lu}$ et al. 2019), and digital twin-driven smart manufacturing (Lu et al. 2020).

In summary, domain knowledge representation has made some progress, especially in the knowledge schema modeling. In-process data will generate in-process information and knowledge. However, most of the current knowledge systems do not have the feature of converting raw data into useful knowledge. Namely, the knowledge base cannot manage in-process data while the database cannot save intricate knowledge.

\section{Dataspace and industrial dataspace}

Dataspaces was first introduced in the informatics as a novel abstraction for data management (Franklin et al. 2005).
Modeling of data, integrating resource entities, discovering relations of resource entities, and indexing of information have been separately studied (Mirza et al. 2010; Niinimaki and Thanisch 2019). In the context of manufacturing, industrial dataspace was regarded as a broker to run CPS by mediating between bottom manufacturing data and upper software (Monostori et al. 2016). In the meantime, the International Data Spaces was proposed to create a secure data space that supports enterprises of different industries and different sizes in the autonomous management of data (Pullmann et al. 2017; Ahmadian et al. 2018). The Industrial Data Space can be seen as a manufacturing data management system in the cross-enterprise level (Angrish et al. 2017). In industrial applications, Ontology-Based Data Access and Knowledge Graph were used to integrate, store, index, semantically query, and knowledge reasoning (Xiao et al. 2018). GE Co. proposed a semantic-driven framework SemTK, which can realize tried-and-true storage and access to heterogeneous data. Moreover, the Polystore KGs with SemTK, which is good at constructing and querying, were employed in four industrial cases (McHugh et al. 2017).

In summary, the terms Dataspace and Data Space are already defined in the literature, and its description methods, structures, and functions are involved. However, the above dataspaces are usually used for managing knowledge in the level of enterprise and cross-enterprise, while there is less research on dataspace in the workshop level. Furthermore, managing heterogeneous data, interconnecting the resource entities and handling domain knowledge are urgently needed in the dataspace of workshop level.

\section{Construction of the IDMW}

From "Problems to be solved and critical review of related work" section, it can be seen that research on machining in-process data, industrial database, manufacturing dataspace has been carried out whether in literature or industrial practices. Nevertheless, there is still no dataspace building for the machining workshop specifically. In the meanwhile, the existing dataspaces usually focus on the data administration in the field of information technology, considering less about manufacturing logics and machining mechanisms. There are no clear ways yet on how to transform machining in-process data into reusable knowledge. Besides, how to discover the links between knowledge and raw data is also unclear. Evidently, an ideal IDMW should not only realize data management but also discover hidden relations among resource entities. Thus, setting up a proper framework of IDMW can facilitate heterogeneous data storage and machining domain knowledge handling. 


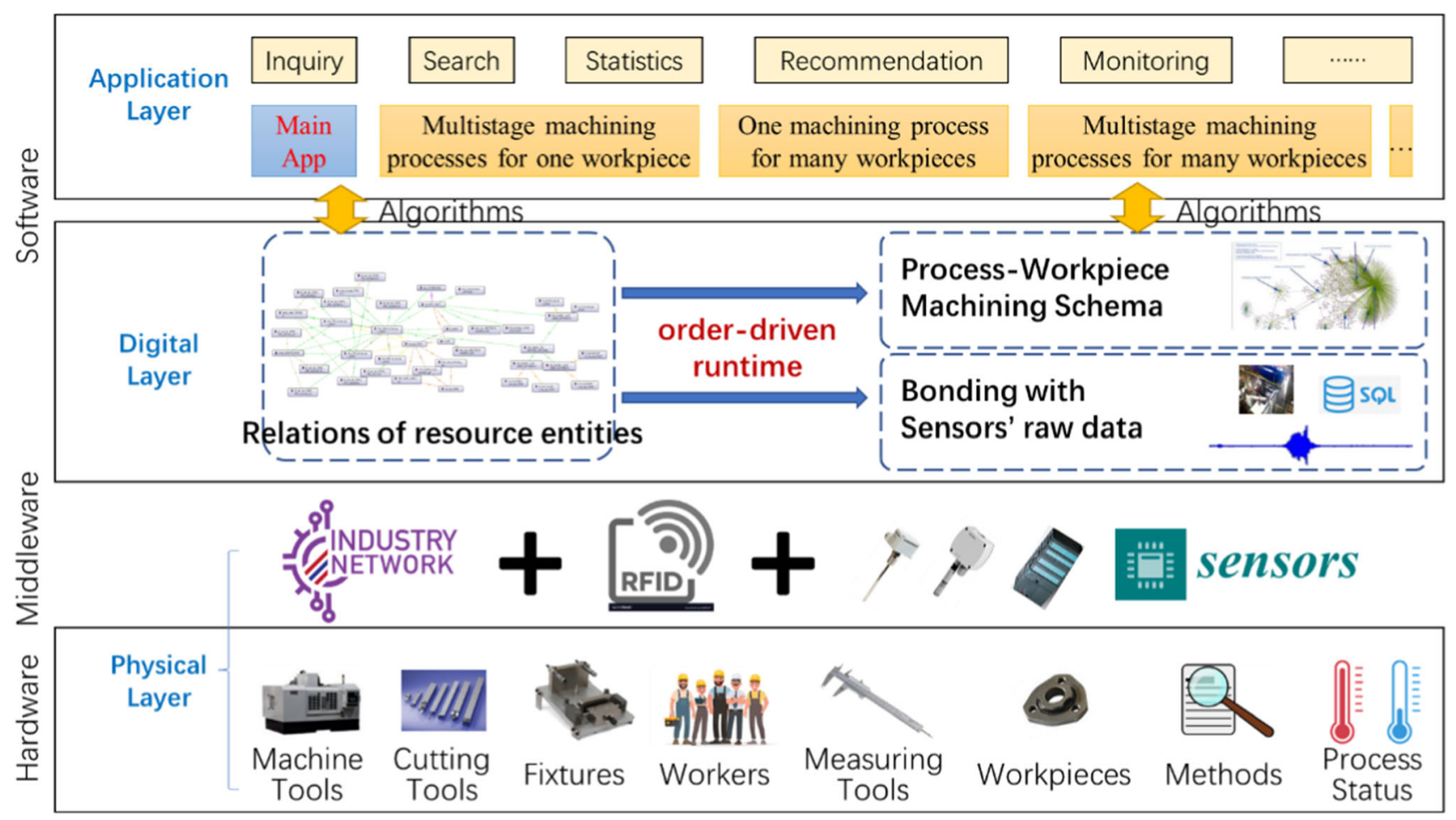

Fig. 2 Framework for industrial dataspace for the machining workshop

\section{Framework of IDMW}

IDMW is a complicated and high-dimensional space, including lots of resource entities, data, and knowledge. As Fig. 2 shows, IDMW can be divided into three parts from the machining roles they played, i.e., hardware, middleware, and software. Hardware refers to the MOC-associated resource entities, mainly including the Machine Tools, Cutting Tools, Fixtures, Workers, Measuring Tools, Workpieces, Machining Methods, and Machining Process Status. Software contains all the industrial applications (APP), systems, software, databases, and knowledge bases that support the MOC. Middleware is the intermediary of Hardware and Software, which is composed of networks, sensors, and RFID devices.

All hardware and middleware resource entities can be regarded as the Physical Layer in IDMW. The other two are Application Layer and Digital Layer, which all belong to the Software part. In Application Layer, there is a leading application portal and several pre-set machining scenarios, each of which is supported by a kind of algorithm. For instance, the model of machining error propagation network (MEPN) (Li and Jiang 2017) can be employed when dealing with the multistage machining processes for one-of-a-kind production. Besides, Application Layer offers interfaces for users with various functions, such as inquiry, search, recommendation, monitoring. Machining domain knowledge, in the form of an algorithm or a model, can be viewed as knowledge schema in
Digital Layer. Furthermore, Digital Layer will manage data collected from the CPS in middleware.

From the view of the application, the Physical Layer in IDMW is mainly for data sensing and collecting, which is the prerequisite work for currying on the utilization of domain knowledge. Digital Layer can be subdivided into two levels, which are the entities' relation storage based on the knowledge schema, and the data management based on the structured query language (SQL) relational database technology. Furthermore, the machining-related knowledge and several decentralized databases are in the Digital Layer, which will support the multiple usages of knowledge in the supreme level, i.e., the Application Layer. Thus, the architecture of Schema Centralized-Data Distributed has been formed in IDMW.

\section{Process-workpiece-centric relation schema description in IDMW}

The uniform schema is employed to describe the relations of machining-related resource entities (Jiang et al. 2019). Existing standards and modeling languages, such as ISA-88/95, $O W L 2$, AutomationML, and $U M L$, could help to define the concepts of meta-data model such as vocabularies, terms, elements, components (Vogt et al. 2019). In the context of manufacturing, ontologies are used to capture domain knowledge. There are many ways to design hierarchy classes during 


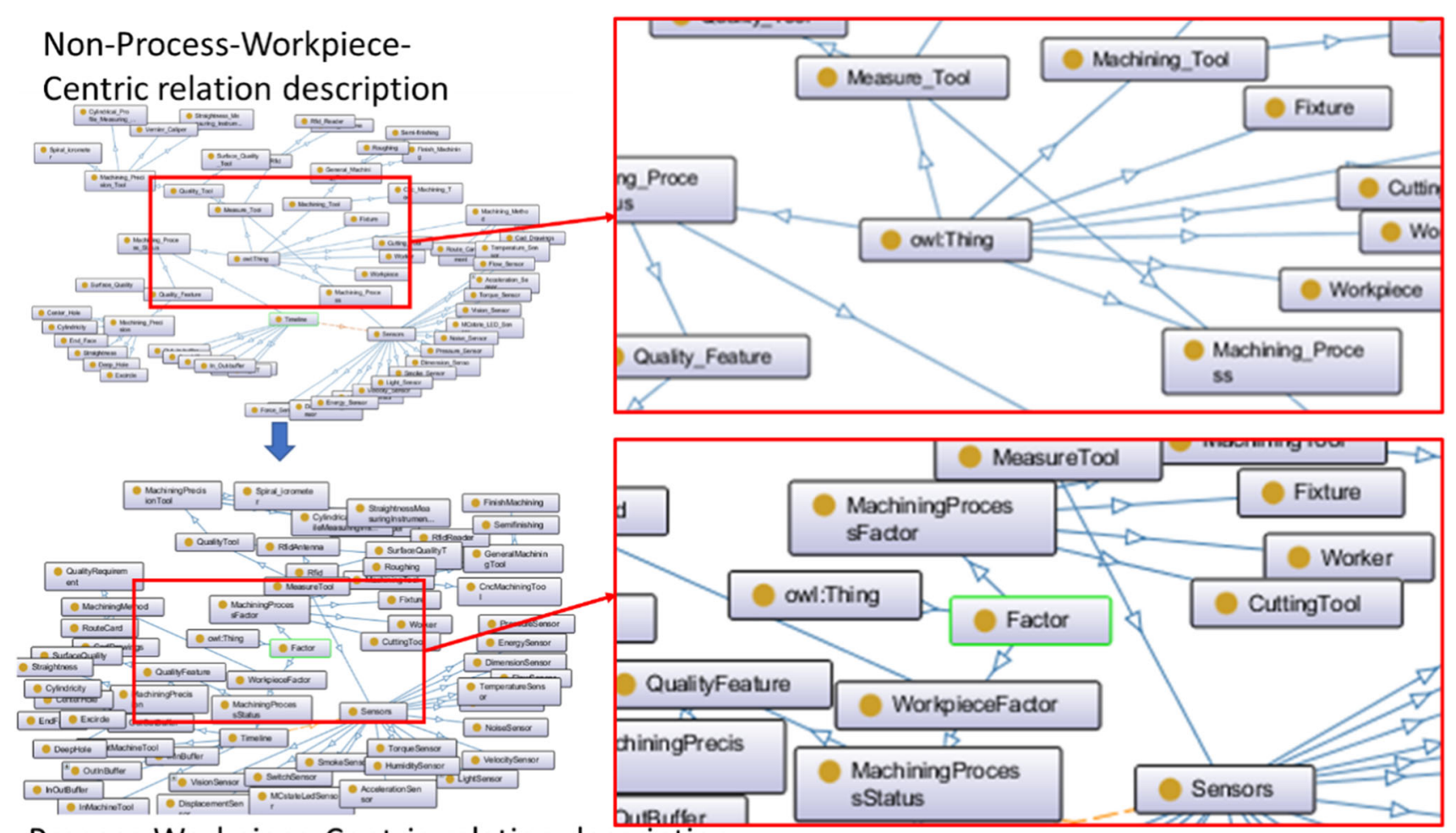

Process-Workpiece-Centric relation description

Fig. 3 Differences between non-process-workpiece-centric and the proposed process-workpiece-centric schemas

ontology development. The conventional method is to classify resources according to their physical characteristics. Since the intelligent machining can be seen as the process that a smart workpiece is processed a smart machining workstation, the main resource entities are machining process and workpiece. Hence, a Process-Workpiece-Centric relation descriptive schema is invented. Moreover, the Protégé tool is employed for ontology modeling. Differences between a conventional relation schema description (Liu et al. 2020) and the proposed Process-Workpiece-Centric one can be seen in Fig. 3. The Process-Workpiece-Centric schema takes the MachiningProcessFactor and WorkpieceFactor as two main subclasses of Factor, which is more suitable for intelligent machining. Creating the standardized description model is to provide a unified way to represent relations of resources entities in machining workshops.

Each resource entity in the workshop has its own uniform resource identifier (URI). For example, a worker $w k_{-} 4115001008$ measures the machining quality feature $q f \_w p \_\lg r \_0245 \_280 \_c y l i n d r i c i t y$ of the workpiece $w p_{-} \lg r \_0245$, with a measuring tool $m s t q \_w a l e_{-} R S 1600$ in the machining process $m p \_280 \_o f \_w p \_\lg r \_0245$. It can be written with non-Process-Workpiece-Centric relation description

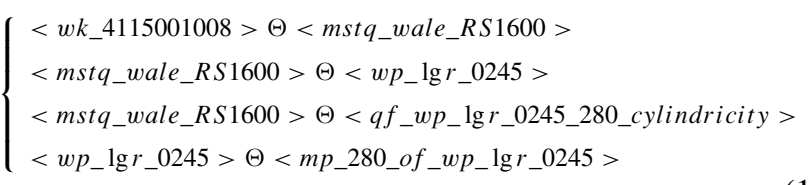

It can also be represented with Process-Workpiece-Centric relation description

$\left\{\begin{array}{l}<w p \_l g r \_0245>\Theta<m p \_280 \_o f \_w p \_\lg r \_0245> \\ \left\{w p \_l g r \_0245>\Theta<q f \_w p \_\lg r \_0245 \_280 \_c y l i n d r i c i t y>\right. \\ <m p \_280 \_o f \_w p \_\lg r \_0245>\Theta<w k \_4115001008> \\ <m p \_280 \_o f \_w p \_\lg r \_0245>\Theta<\text { mstq_wale_RS } 1600>\end{array}\right.$

In the second description, the primary relation, that is, the Process-Workpiece relation, will be identified preferentially, which contributes to find the relations among distributed Individuals quickly. From the perspective of a single process in a workstation, all its machined workpieces can be quickly associated. From the angle of the machining process flow of a workpiece, its machining process can be connected in series easily.

In the Process-Workpiece-Centric descriptive schema, all the non-core resource entities can be linked to whether MachiningProcessFactor or WorkpieceFactor. Individuals of MachiningProcessFactor are the virtual processes that make up the machining process flow, and the Individuals 


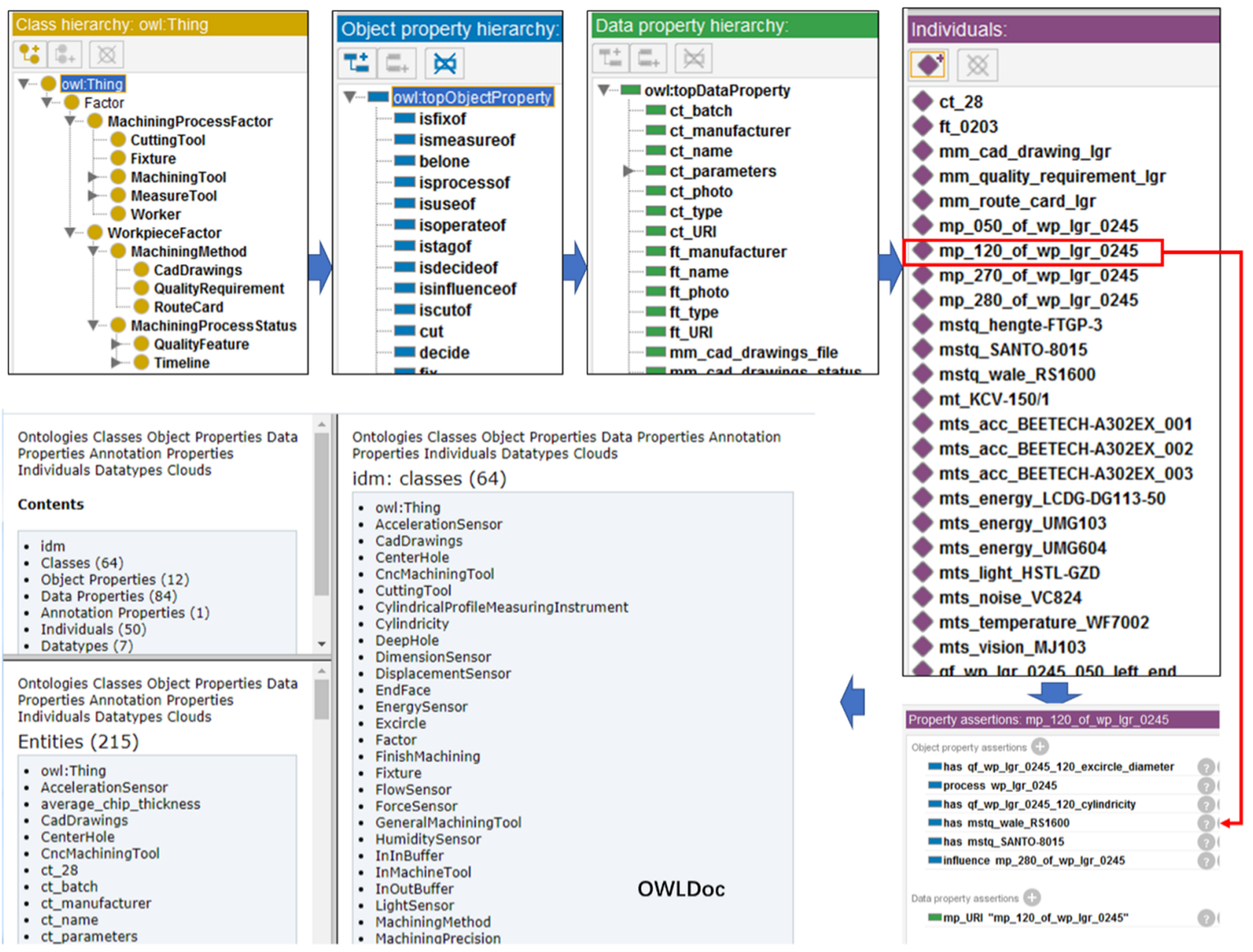

Fig. 4 The ontology development process for a process-workpiece-centric schema

of WorkpieceFactor are smart workpieces. For example, a framework of resources followed by the $\mathrm{W} 3 \mathrm{C}$ web ontology language (OWL) standard can be seen in Fig. 4, where CuttingTool, Fixture, MachiningTool, MeasuringTool, and Worker are subclasses of MachiningProcessFactor, and MachiningMethod, MachiningProcessStatus are subclasses of WorkpieceFactor.

\section{Distributed data storage in digital layer based on process-workpiece-centric schema}

Then, the distributed data are linked to the entities in the prefabricated Process-Workpiece-Centric schema. Although the raw machining data can be directly stored as Object Properties or Data Properties of Individuals, they are still designed to be stored apart of the schema as multiple data silos.

The Schema Centralized-Data Distributed architecture can make the operation of IDMW more secure and robust. On the one hand, the instantaneous data generated by the RFID change the status of the resource entities. This kind of change will modify the Data Properties of Individuals, where these data are not very big in scale. On the other hand, data generated by the sensors are generally with features of massive, high-dimensional, heterogeneous, and distributed. As Fig. 5 shows, vision sensors generate a series of monitoring video files (.mp4), acceleration sensors create massive continuous data files (.mat), and temperature sensors offer discrete serialized sampling data saved in the SQL relational database (.sql). These kinds of data are continuously generated as long as the workshop runs, but they are with limited information before being associated with the Process-Workpiece-Centric schema.

\section{Pre-processing of the data silos during the runtime of IDMW}

The runtime of IDMW can be divided into three major procedures mainly, which are Determination of timelines driven 


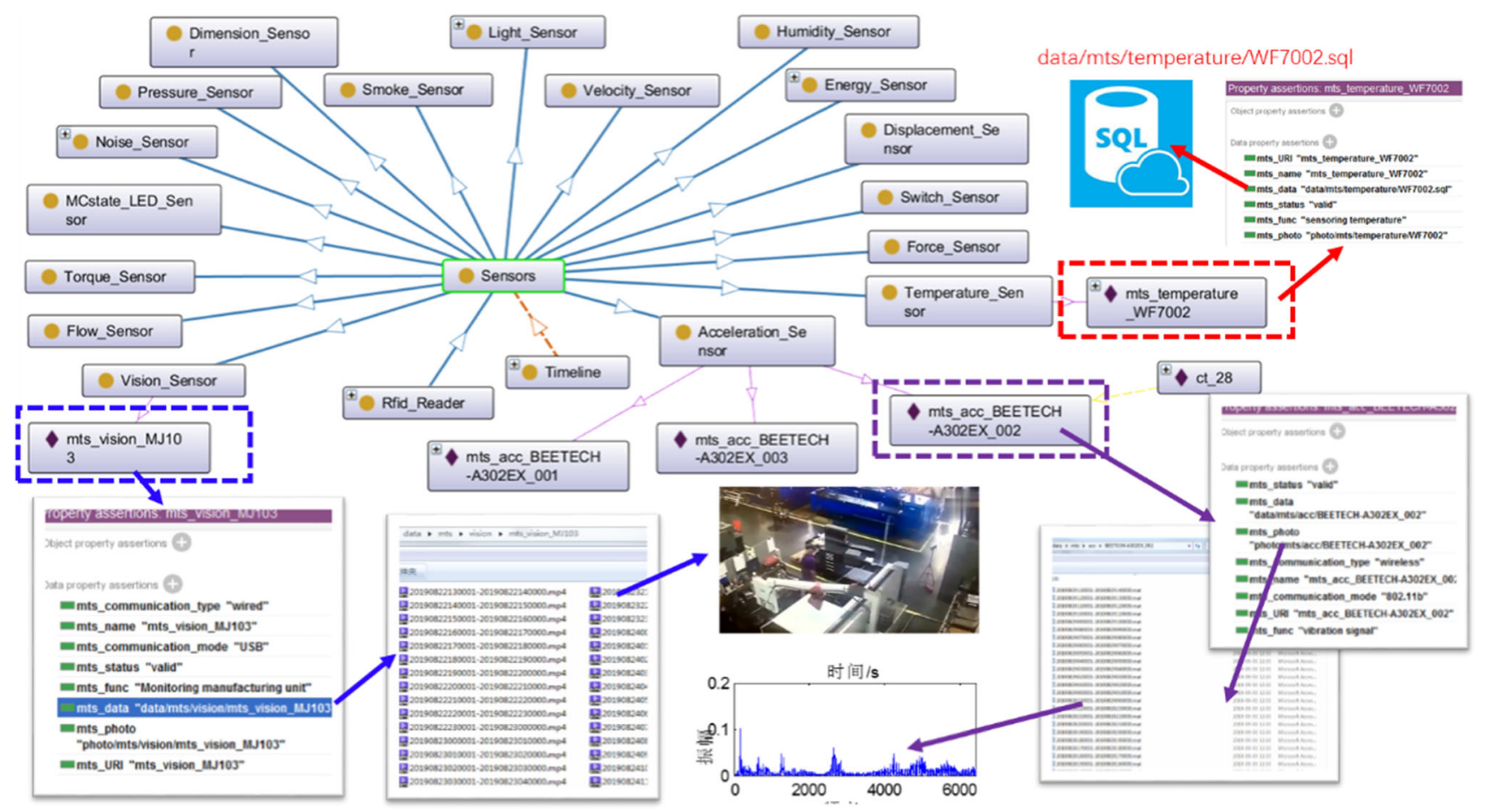

Fig. 5 Different kinds of data from distributed sensors associated by knowledge schema

by RFID event graphical deduction model, Pre-processing of disparate data silos, and Reuse of domain knowledge.

\section{Determination of timelines driven by RFID event graphical deduction model}

The most important two Classes in Process-WorkpieceCentric schema is MachiningProcessFactor and WorkpieceFactor, which needs to be determined first. In intelligent machining, smart workpieces will be delivered to the machining workshop after the order was placed. Then, the Gantt Chart will be generated and dispatched as machining planning. At this point, the binding between workpieces (WorkpieceFactor) and processes (MachiningProcessFactor) has accomplished.

In order to find the specific starting and ending time in a machining process, the events graphical deduction model (Wang et al. 2018) is employed for monitoring the real-time machining. Some definitions are defined as follows:

Definition 1 Timelines are defined as a series of virtual baselines with the same timestamp while machining. Some specific timelines were selected as the machining events, which are the actions that happen on processes, such as machining starting event, machining ending event. Timelines are determined by both the RFID readers/antennas configured in the processes and the RFID tags inside workpieces, which shown in Fig. 6. These timelines, or the machining event, can be formalized by the following equation

$t_{P}^{x}(x=s, s i, e i, s p, e p, s o, e o, e)$

where $t_{P}^{s}$-Arriving Timeline from the last process to the current process (in RFID antenna 1). $t_{P}^{s i}$-Starting Timeline of loading workpiece to machine tool table (out RFID antenna 1). $t_{P}^{e i}$-Ending Timeline of loading workpiece in machine tool table (in RFID antenna 2). $t_{P}^{s p}$ - Starting Timeline of processing workpiece (starting of machine tool). $t_{P}^{e p}$-Ending Timeline of processing workpiece (ending of machine tool). $t_{P}^{s o}$ - Starting Timeline of putting workpiece off machine tool table (out RFID antenna 2). $t_{P}^{e o}$-Ending Timeline of putting workpiece off machine tool table (in RFID antenna 3). $t_{P}^{e}$-Leaving Timeline from the current process (out RFID antenna 3 ).

Definition 2 Machining states are defined as the continuous status between two consecutive machining events, such as workpiece-in-in-buffer, workpiece-in-machining, workpiece-in-inspecting, workpiece-in-out-buffer. Usually, a machining event would trigger the transition from one machining state to another. The machining state can be formalized by

$T_{P}^{x}(x=$ in-buffer,mounting, processing, dismounting,out-buffer $)$ 


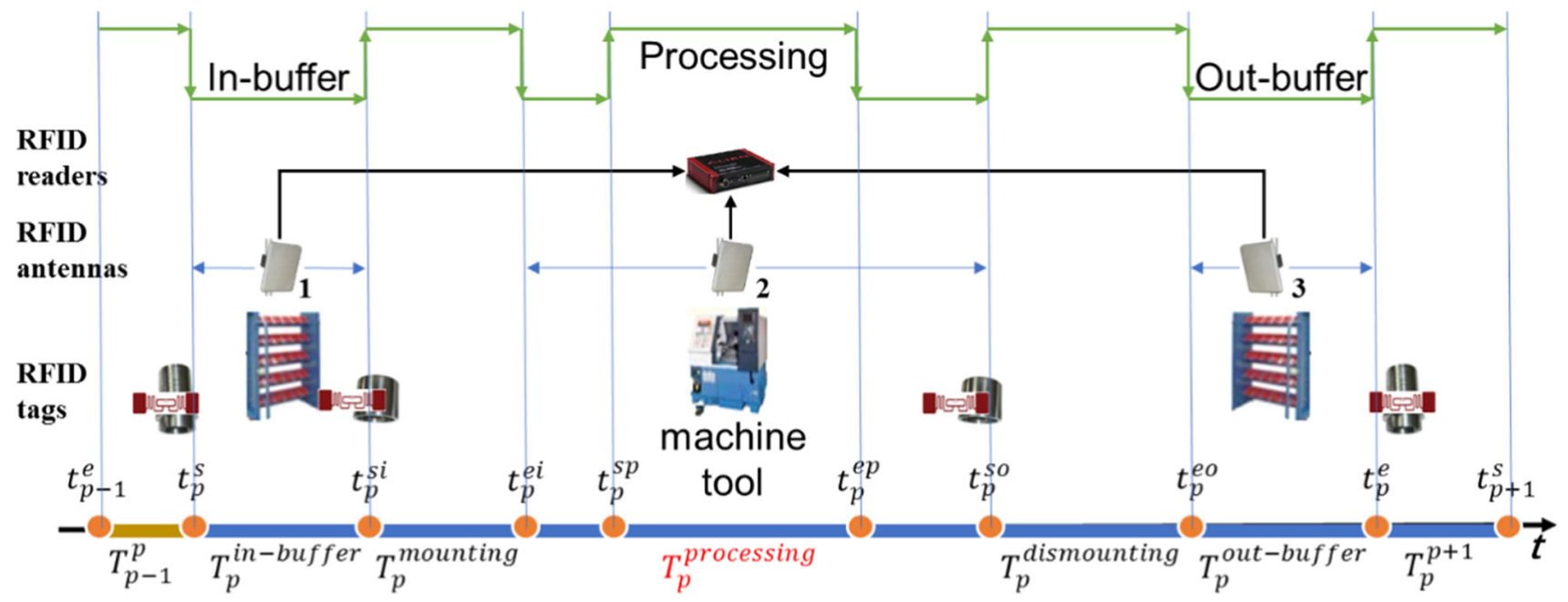

Fig. 6 The RFID event graphical deduction model of the workpiece in one process

where

$$
\begin{aligned}
& T_{P}^{\text {processing }}=\left\{T_{P}^{\text {machining }}\left\|T_{P}^{\text {inspecting }}\right\| T_{P}^{\text {heat } 0 \text { treating }} \|\right. \\
& \left.T_{P}^{\text {benching }}\left\|T_{P}^{\text {surface0treating }}\right\| \ldots\right\} .
\end{aligned}
$$

\section{Pre-processing of disparate data silos}

As mentioned in "Distributed data storage in digital layer based on process-workpiece-centric schema" section, the sensors will continuously generate data in different formats and form several data silos. These data silos need to be preprocessed to pick out the useful data that can characterize the machining process in IDMW. The Timelines that determined by the RFID event graphical deduction model provide a reference time scale for pre-processing these data silos. The loose data in the data silos can be pre-processed as needed to data fragments that reflect machining processes.

As Fig. 7 shows, timelines provide benchmarks for truncating time-series data. Specific to each kind of data silos, the continuous signal such as vibration or energy consumption will be truncated from .mat file within $\left[t_{P}^{s p}, t_{P}^{e p}\right]$, the continuous sampling data such as temperature will be obtained from .sql according to SQL, the vision data will be edited from .$m p 4$, and the photo data such as the latest tool wear will be picked from .jpg at a certain moment. It should be pointed out that only the data silos related to the machining process will be pre-processed, and which data silos will be selected are determined by the Process-Workpiece-Centric schema mentioned in "Process-Workpiece-Centric relation schema description in IDMW" section.

\section{Reuse of domain knowledge}

The goal of IDMW is to realize the application of domain knowledge in the MOC. Therefore, it is necessary to ana- lyze the interaction among various resource entities during the runtime of IDMW to illustrate how domain knowledge is expressed and applied. Figure 8 shows the interaction of several resource entities during the runtime of IDMW with a multi-role swim lane diagram. The interaction-based instructions are detailly illustrated in Table 1.

The domain knowledge here is mainly reflected in two aspects, namely the machining pattern determination in 503 and the implementation algorithm application in $s 033$. The machining pattern is stored in the Application Layer, which can be used to determine which kinds of domain knowledge to be used. The implementation algorithms are composed of data analyzing models, such as the support vector machine model and the correlation analysis model, which can be used for the MOC process.

\section{Industrial case studies}

This section reports two real-world industrial cases on IDMW based MOC in machining workshops. The first case is from a landing gear manufacturer whose production is small-batch and multi-process, and the goal is to evaluate the stability of the machining process using the IDMW. The second one comes from a precision machining workshop whose aim is achieving reliable quality prediction of precision $\mathrm{CNC}$ with the help of the IDMW. In these two cases, the simpler and small data are marked as data properties of Individuals in the schema while the numerous data are stored in separate data silos. The schema is centralized while the data silos ack as decentralized nodes. The architecture of Schema Centralized-Data Distributed helps to realize the better performance on efficiency and safety. 


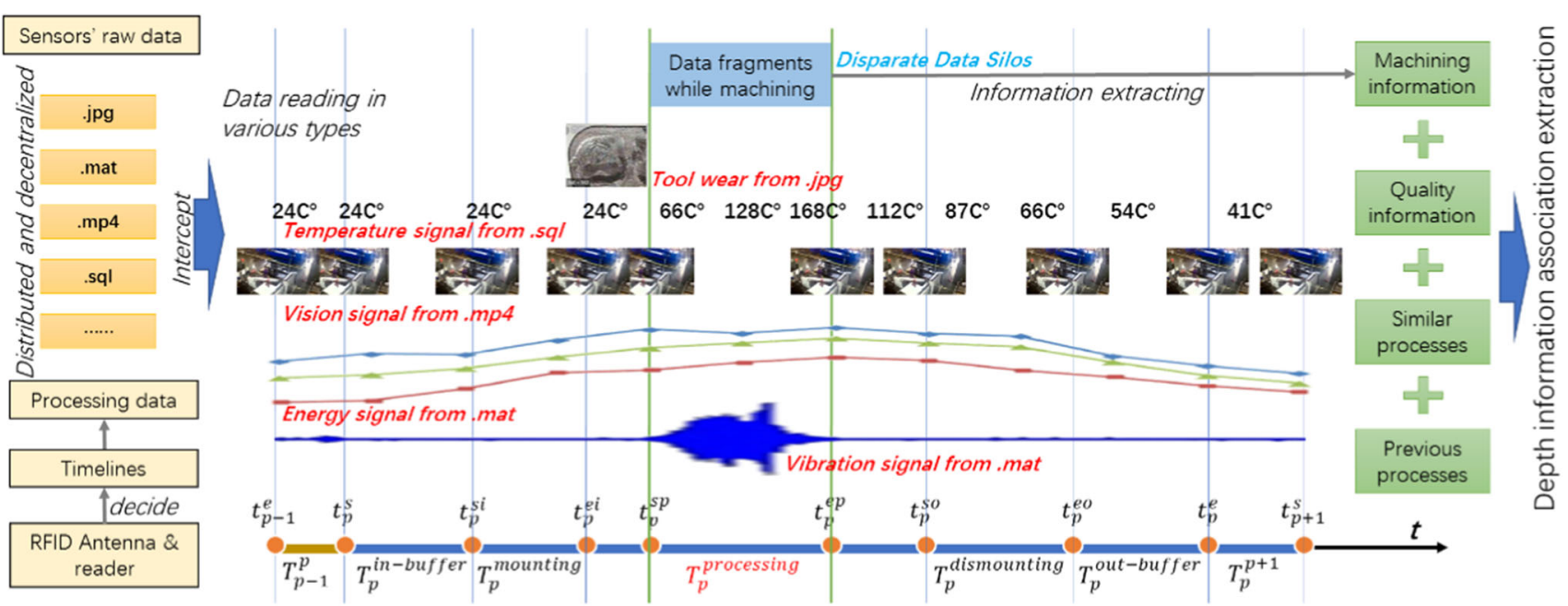

Fig. 7 Pre-processing of disparate data silos within a machining process

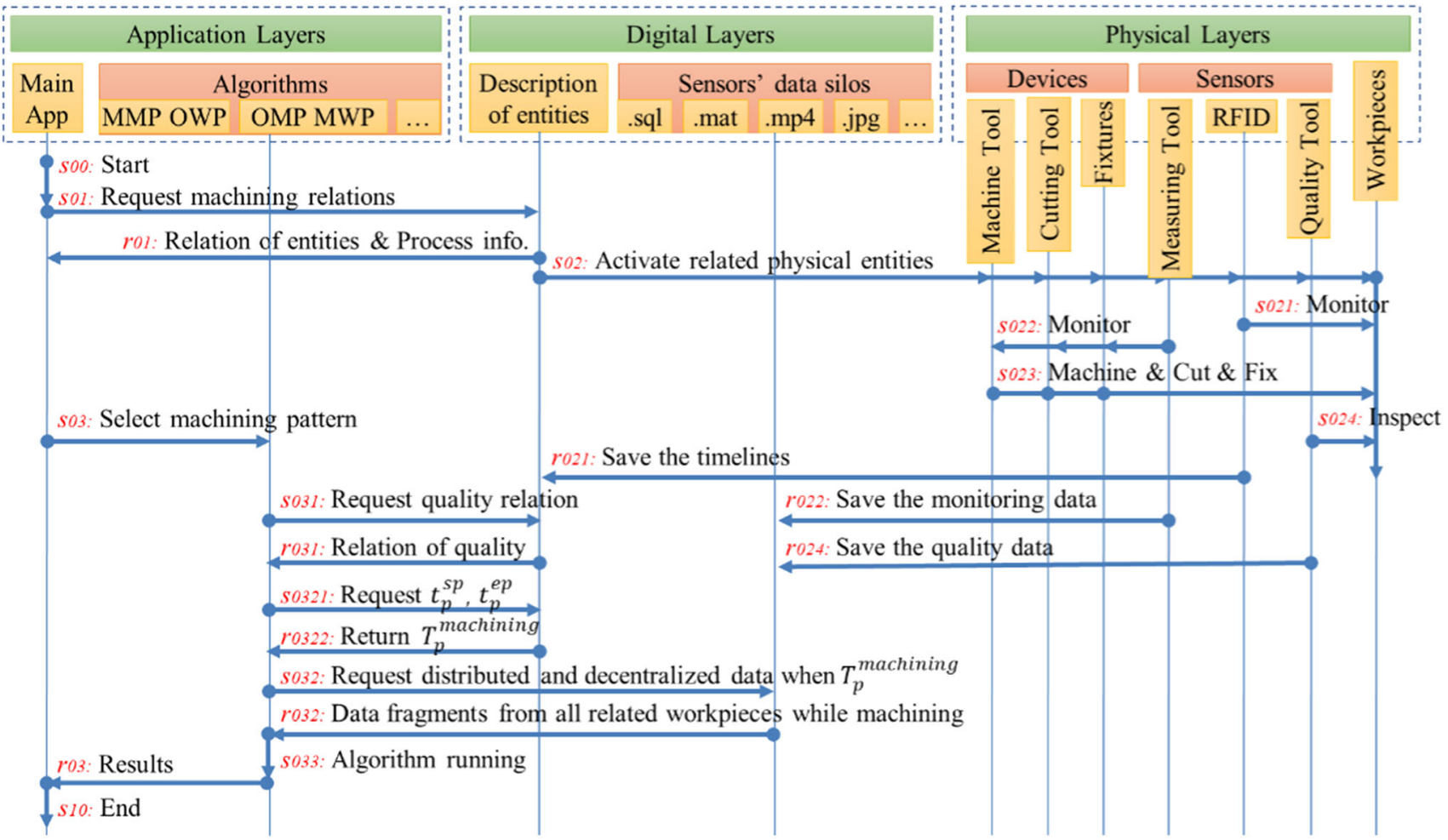

Fig. 8 The interaction of several resource entities during the runtime of IDMW with a multi-role swim lane diagram

\section{Case study I: Using MEPN knowledge in IDMW to evaluate the machining stability}

The products are Landing Gear Main Cylinder (lgr_0245), which has five-stage deep-hole machining processes. As "Construction of the IDMW" section mentions, ProcessWorkpiece-Centric schema for the whole workshop needs to be modeled firstly, and then use the domain knowl- edge (MEPN and stability evaluation model) to evaluate the machining stability.

\section{Modelling of the IDMW}

Aircraft trial Workpiece lgr_0245 has 40 machining processes, and it is one-of-a-kind production. Thus, a total of 41 individuals are extracted as main entities, including 40 individuals of MachiningProcessFactor ( $m p \_x x x \_o f \_l g r \_0245$ ) 
Table 1 The detail interaction-based instructions during the runtime of the IDMW

\begin{tabular}{|c|c|c|c|c|c|}
\hline \multirow{2}{*}{$\begin{array}{l}\text { No. } \\
\text { s00 }\end{array}$} & & & \multirow{2}{*}{$\begin{array}{l}\text { Description } \\
\text { Start }\end{array}$} & \multirow{2}{*}{$\begin{array}{l}\text { Results } \\
\text { s01 }\end{array}$} & \multirow[t]{2}{*}{ Memo } \\
\hline & & & & & \\
\hline s01 & & & Request machining relations & r01 and s02 & Traverse all relations \\
\hline$r 01$ & & & Relation of entities and process info & s03 & \\
\hline \multirow[t]{8}{*}{ s02 } & & & Activate related physical entities & s021 and s022 and s023 and s024 & Start machining \\
\hline & $s 021$ & & Monitor & r021 & \\
\hline & $r 021$ & & Save the timelines & & Store as property of instance \\
\hline & s022 & & Monitor & r022 & \\
\hline & r022 & & Save the monitoring data & & Generate data silos \\
\hline & s023 & & Machine and cut and fix & & \\
\hline & s024 & & Inspect & r024 & Generate quality data \\
\hline & r024 & & Save the quality data & & \\
\hline \multirow[t]{8}{*}{ s03 } & & & Select machining pattern & s031 and s032 and s033 & $\begin{array}{l}\text { Multistage machining processes for } \\
\text { one workpiece, One process for } \\
\text { Multi workpiece, One process for } \\
\text { one workpiece, ... }\end{array}$ \\
\hline & s031 & & Request quality relation & r031 & \\
\hline & r031 & & Relation of quality & s033 & For algorithms \\
\hline & & $s 0321$ & Request $t_{P}^{s p}, t_{P}^{e p}$ & $r 0322$ & \\
\hline & & r0322 & Return $T_{P}^{\text {machining }}$ & $s 032$ & \\
\hline & s032 & & $\begin{array}{l}\text { Request distributed and decentralized } \\
\text { data when } T_{P}^{\text {machining }}\end{array}$ & r032 & \\
\hline & $r 032$ & & $\begin{array}{l}\text { Data fragments from all related } \\
\text { workpieces while machining }\end{array}$ & s033 & For algorithms \\
\hline & s033 & & Algorithm running & $r 03$ & Use domain knowledge \\
\hline r03 & & & Results & & \\
\hline s10 & & & End & & \\
\hline
\end{tabular}

and an individual of WorkpieceFactor. After associating with all the related Individuals of MachineTools, CuttingTools, Fixtures, Workers, MeasuringTools, MachiningMethods, and MachiningProcessStatus, the MEPN is used to identify the key process that affects the final quality most (Li and Jiang 2019). Result shows that the individual $m p \_280 \_o f \_w p \_l g r \_0245$ is the key process, and individuals mp_050_of_wp_lgr_0245, mp_120_of_wp_lgr_0245 and $m p \_270 \_o f \_w p_{-} \lg r \_-\overline{0} 45$ are three processes that affect the key process. Subsequently, the stability evaluation model is employed to figure out the status of the key process.

\section{Operation of stability evaluation model in IDMW}

The main Process-Workpiece-Centric schema is shown in Fig. 9, where the colored blocks are the individuals, the white blocks are data properties of individuals.

Instructions $s 00, s 01, r 01$ are executed when constructing the IDMW while $\mathrm{s} 02$ activates related resources entities. Specifically, s021 actives the one rfid_reader and three corresponding rfid_ALIENs to monitor the key process, while r021 returns with six timelines and saves them in the schema. Then, sO22 actives mts acc BEETECHA302EX 002 to monitor ct 28 and ft 0203 , and drive mts temperature_WF7002, mts_acc_BEETECHA302EX 001, mts energy LCDG-DG113-50 to monitor $m t K C V-150 / 1$. The collected raw data in different formats are stored separately in r022. Similarly, s024/r024 get quality data, which are stored in the schema as data properties directly.

s03 is for selecting the machining pattern, which is multistage machining processes for one workpiece. Then, s031/r031 helps to find out the related decentralized data silos while $s 0321 / r 0322$ will pre-process them to data fragments with definite timelines. All the retrieved data are fed to the stability evaluation model, in which the stability index can be calculated. 


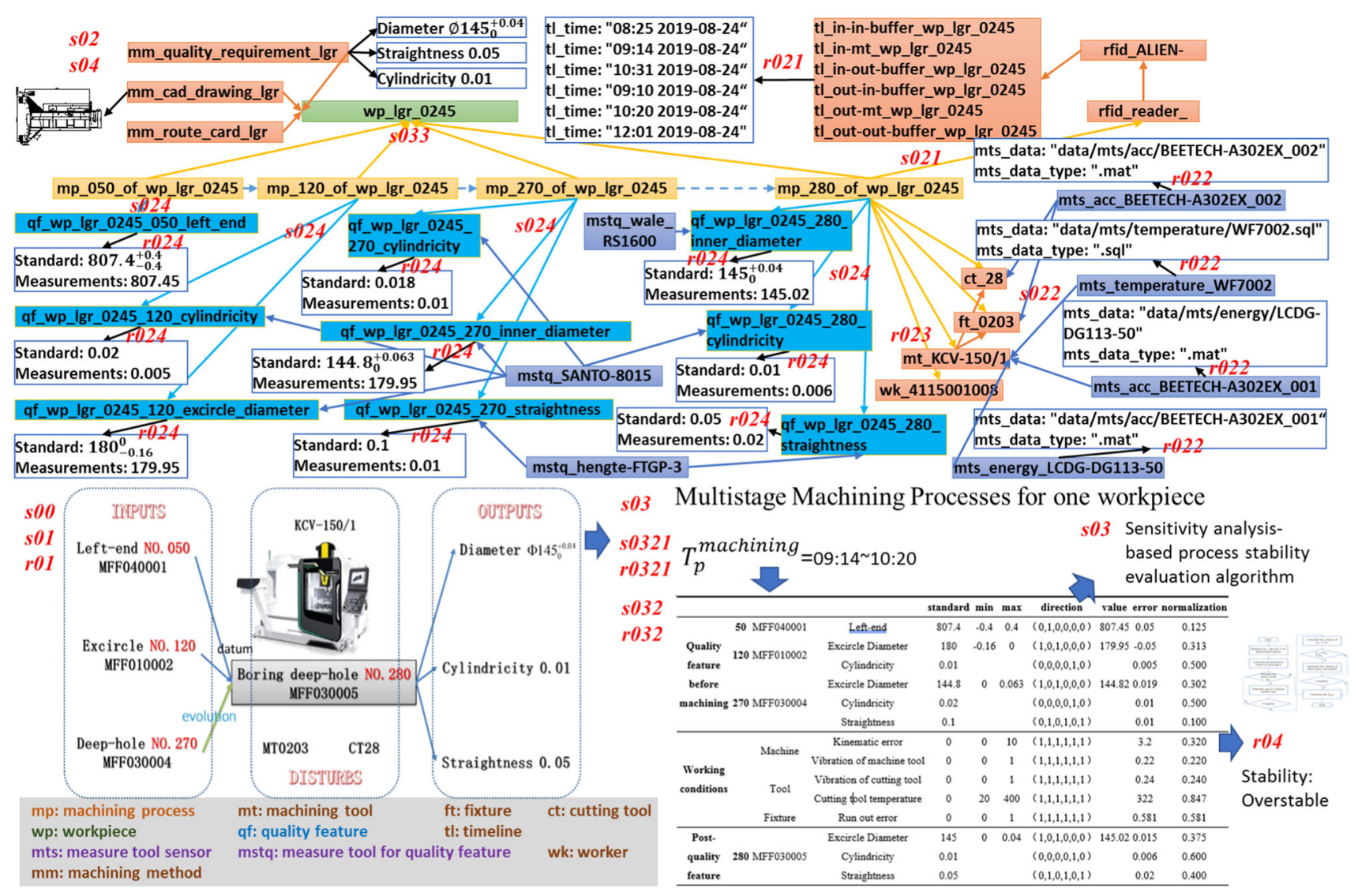

Fig. 9 Using IDMW to evaluate the machining stability of One-of-a-kind production

\section{Case study II: Using the correlation analysis knowledge to predict the precision CNC machining quality of multiple workpieces}

Case I focused on the application flow of IDMW, while Case II pays attention to the handling and reuse of domain knowledge.

The wear of tools, ambient temperature, and energy consumption will affect the final machining quality performance when precision machining. Thus, a series of machining experiments have been conducted to find mathematical linking in the Brunel University London Advanced Manufacturing Laboratory, which can be viewed as a precision machining workshop (Katchasuwanmanee et al. 2015). Then IDMW is used for data collection, data analysis, knowledge storage, and knowledge reuse.

\section{Construction of the IDMW}

IDMW in Case II is mainly for the efficient storage of machining in-process data, information, and knowledge. Thus, it is needed to model the relations of resource entities first and foremost.
Forty aluminum tests were performed on a $\mathrm{CNC}$ machine at different periods during the days in order to obtain different ambient temperatures of the workshop. All the relations and machining in-process data will be collected and stored in IDMW, which can be seen in Fig. 10. There are forty Individuals of WorkpieceFactor, symbolized from $m p \_e n d-m i l l \_f \_w p \_l$ to $m p \_n d-m i l l \_f \_w p \_40$, and each of them corresponds to two Individuals of MachiningProcessFactor, i.e., $m p \_e n d-m i l l-r o u g h i n g \_o f \_w p \_x$, and $m p \_e n d-m i l l-f i n i s h i n g \_o f \_w p \_x$. The IDMW can document all the machining data from sensors.

\section{Reuse of domain knowledge stored in IDMW}

Supposing that there comes a new workpiece $m p \_$endmill of wp test to be machined. It has two machining processes that are $m p \_$end-mill-roughing_of_wp_test with cutting tool $c t$ SSGS-roughing-Tool, and $m p \_e n d-m i l l-$ finishing_of_wp_test with cutting tool ct SGS-finishingTool. The machine tool mt_cnc_KERN-5-Axis-HSPC-2216 and machining method $m_{-}$machining parameters are also given.

In the beginning, a relational query is executed to retrieve all similar historical individuals of MachiningProcessFac- 


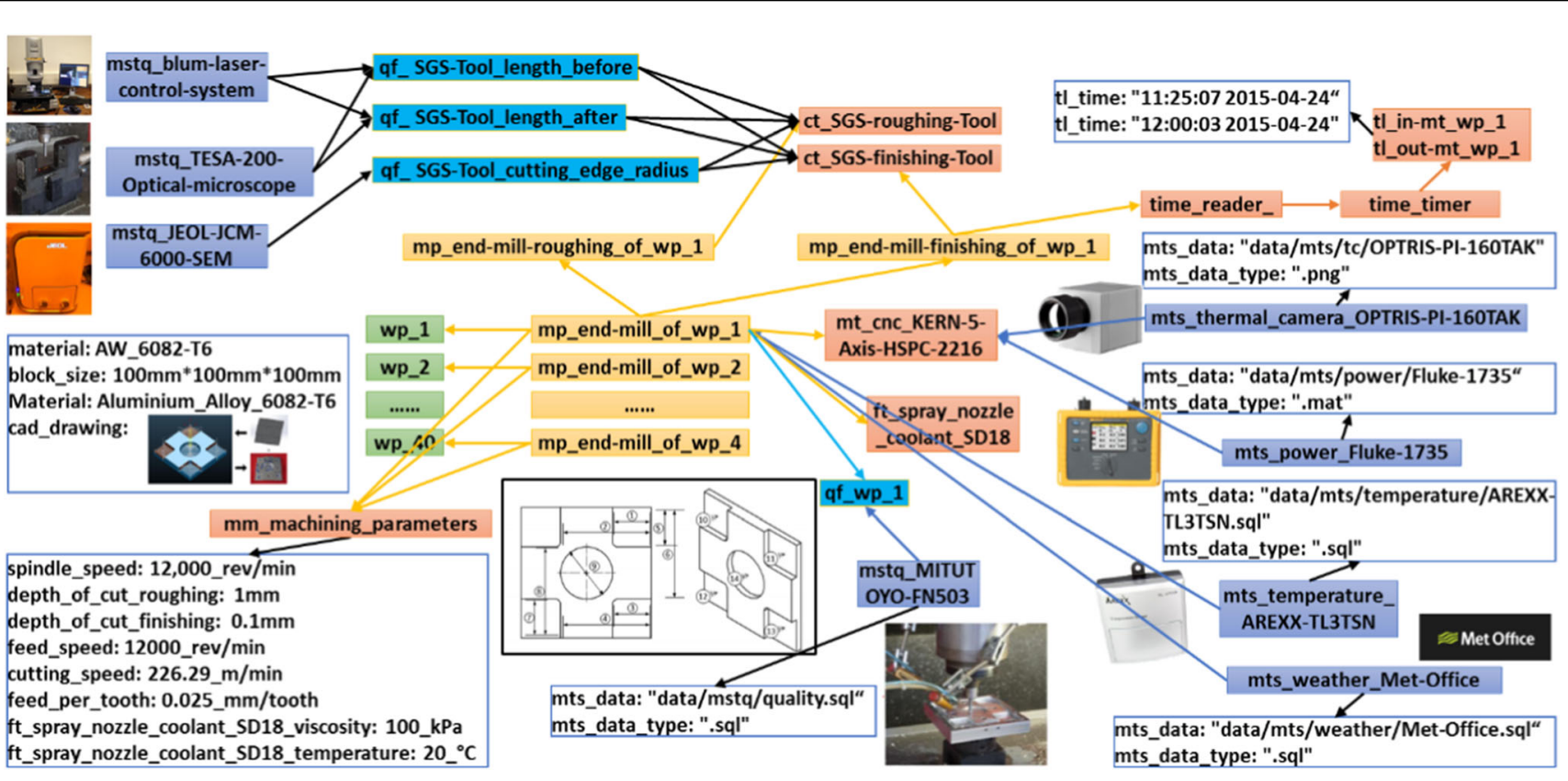

Fig. 10 Using IDMW to predict precision machining quality of multiple workpieces

tor (with the same Individuals of MachineTool, CuttingTool, Fixture). The SPARQL Query Language is shown as follows. energy consumption $(\mathrm{kWh})$ and ambient temperature $\left({ }^{\circ} \mathrm{C}\right)$ is described with

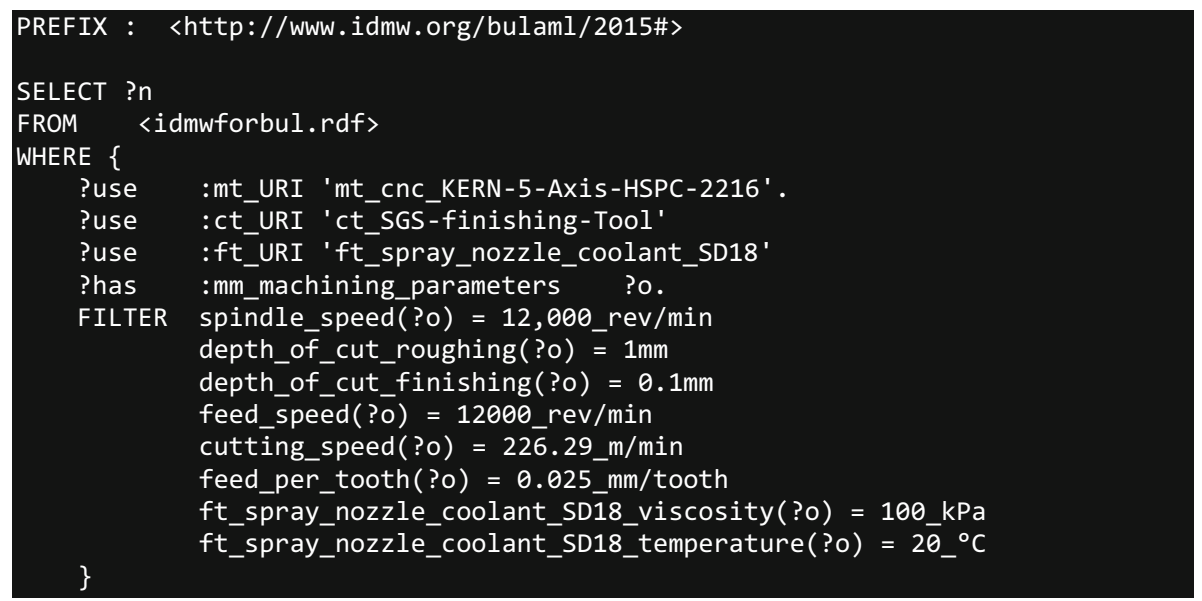

With that, all the previous 40 workpieces (Individuals) can be found out, and the timelines of these 40 workpieces are also separately requested. Then, the relevant data can be taken out from distributed data silos, which shows in Table 2.

At this point, an algorithm of linear regression analysis was performed in the Application Layer. Results show that the quality of the workpiece is degraded as the temperature increases, and a negative correlation can be found between ambient temperature and energy consumption. The three-dimensional correlation of quality error (\%), total where $z$ is the quality error; $x$ is the ambient temperature, and $y$ is the total energy consumption. Results indicate that the ambient temperature should be monitored and controlled accordingly to achieve product quality standards and also to reduce energy usage. Additionally, the quality of the current workpiece can be predicted by the fitted regression equation. Moreover, the processing data of the new workpiece will be also stored in the IDMW. 
Table 2 Relevant data of previous 40 workpieces taken from IDMW

\begin{tabular}{|c|c|c|c|c|c|c|c|}
\hline Wp (no.) & $\begin{array}{l}\text { Ambient } \\
\text { temperature } \\
\left({ }^{\circ} \mathrm{C}\right)\end{array}$ & $\begin{array}{l}\text { Total energy } \\
\text { consumption } \\
(\mathrm{kWh})\end{array}$ & $\begin{array}{l}\text { Quality error } \\
(\%)\end{array}$ & $\begin{array}{l}\text { Tool } \\
\text { wear-roughing } \\
(\mu \mathrm{m})\end{array}$ & $\begin{array}{l}\text { Tool cutting } \\
\text { edge } \\
\text { radius-roughing } \\
(\mu \mathrm{m})\end{array}$ & $\begin{array}{l}\text { Tool } \\
\text { wear-finishing } \\
(\mu \mathrm{m})\end{array}$ & $\begin{array}{l}\text { Tool cutting } \\
\text { edge } \\
\text { radius-finishing } \\
(\mu \mathrm{m})\end{array}$ \\
\hline 1 & 23.6 & 11.02 & 0.1452 & 9 & 4.5332 & 4 & 4.3844 \\
\hline 2 & 25.3 & 10.85 & 0.1698 & 15 & & 6 & \\
\hline 3 & 26.1 & 10.76 & 0.1778 & 19 & & 8 & \\
\hline 4 & 25.5 & 10.73 & 0.1836 & 25 & 7.6343 & 9 & 4.8228 \\
\hline 5 & 23.7 & 11.13 & 0.1491 & 30 & & 11 & \\
\hline 6 & 24.3 & 10.93 & 0.1467 & 33 & & 12 & \\
\hline 7 & 26.2 & 10.79 & 0.1907 & 37 & & 13 & \\
\hline 8 & 25.2 & 10.94 & 0.1730 & 39 & 8.9646 & 15 & 5.2087 \\
\hline 9 & 23.9 & 11.05 & 0.1349 & 42 & & 16 & \\
\hline 10 & 24.7 & 10.92 & 0.1636 & 46 & & 18 & \\
\hline 11 & 26.5 & 10.69 & 0.1971 & 49 & & 19 & \\
\hline 12 & 25.9 & 10.79 & 0.1828 & 53 & 9.4382 & 21 & 5.5889 \\
\hline 13 & 23.3 & 11.32 & 0.1466 & 56 & & 23 & \\
\hline 14 & 25.7 & 10.86 & 0.1746 & 58 & & 24 & \\
\hline 15 & 26.3 & 10.75 & 0.1960 & 60 & & 25 & \\
\hline 16 & 26.6 & 10.67 & 0.1990 & 61 & 10.1692 & 26 & 6.3713 \\
\hline 17 & 23.8 & 11.24 & 0.1475 & 63 & & 27 & \\
\hline 18 & 25.0 & 10.99 & 0.1617 & 66 & & 28 & \\
\hline 19 & 25.6 & 10.86 & 0.1860 & 68 & & 30 & \\
\hline 20 & 25.8 & 10.74 & 0.1723 & 71 & 11.9811 & 31 & 6.4988 \\
\hline 21 & 23.4 & 11.27 & 0.1476 & 73 & & 33 & \\
\hline 22 & 26.5 & 10.57 & 0.2122 & 75 & & 34 & \\
\hline 23 & 25.8 & 10.66 & 0.2072 & 76 & & 36 & \\
\hline 24 & 25.7 & 10.81 & 0.1894 & 78 & 13.4812 & 37 & 7.2136 \\
\hline 25 & 23.8 & 11.12 & 0.1391 & 79 & & 39 & \\
\hline 26 & 25.4 & 10.80 & 0.1762 & 81 & & 40 & \\
\hline 27 & 26.3 & 10.67 & 0.1923 & 84 & & 41 & \\
\hline 28 & 25.4 & 10.92 & 0.1877 & 86 & 14.6927 & 43 & 8.3678 \\
\hline 29 & 23.9 & 11.21 & 0.1494 & 88 & & 45 & \\
\hline 30 & 25.8 & 10.84 & 0.1792 & 91 & & 46 & \\
\hline 31 & 26.1 & 10.71 & 0.2060 & 93 & & 47 & \\
\hline 32 & 26.8 & 10.57 & 0.2060 & 96 & 15.9731 & 48 & 9.6230 \\
\hline 33 & 24.1 & 11.20 & 0.1516 & 98 & & 49 & \\
\hline 34 & 25.3 & 10.74 & 0.1880 & 101 & & 51 & \\
\hline 35 & 25.3 & 10.97 & 0.1681 & 103 & & 52 & \\
\hline 36 & 25.9 & 10.67 & 0.2057 & 105 & 18.8214 & 53 & 10.7778 \\
\hline 37 & 24.3 & 11.02 & 0.1580 & 108 & & 55 & \\
\hline 38 & 25.6 & 10.89 & 0.1762 & 111 & & 56 & \\
\hline 39 & 25.4 & 10.93 & 0.1729 & 112 & & 57 & \\
\hline 40 & 26.7 & 10.64 & 0.2027 & 114 & 20.8330 & 58 & 12.8108 \\
\hline
\end{tabular}




\section{Further discussions}

IDMW is designed for collecting all kinds of machining inprocess data, managing them with domain knowledge, and enabling them in MOC. This paper attempts to construct a primary-level IDMW and applies it in some industrial practices. Contributions, technical advantages, and prospects of the proposed IDMW are described separately below.

\section{Contributions of the IDMW}

IDMW mainly has three advance technological contributions.

(1) Accessible for heterogeneous in-process data The novel Schema Centralized-Data Distributed architecture in the Digital Layer makes all in-process data easy to access. IDMW is compatible with all data types, including the multimedia data and sensor data with a variety of formats. Any kind of data can be put into the Digital Layer of IDMW via CPS as long as can be recorded.

(2) Interconnectable of disparate data silos driven by the knowledge schema The knowledge schema in IDMW has the Process-Workpiece-Centric architecture that all the non-core Classes can be linked to either MachiningProcessFactor or WorkpieceFactor. The RFID event graphical deduction model is used to determine the timelines, which can pre-process the disparate data silos, thereby driving the heterogeneous data interconnected.

(3) Flexible storage of domain knowledge The storage of complex machining knowledge in no longer relying on a unified description template. Knowledge in IDMW is represented as relations of resources entities in knowledge schema, and they will not be summarised and represented until needed. In Case II, the knowledge itself (formula $z=0.014 x-0.031 y+0.164$ ) is not stored in IDMW but solved by APP in Application Layer when there is a need for quality prediction of a new workpiece.

\section{Technical advantages of the IDMW}

IDMW shows several significant differences comparing to databases, data warehouses, knowledge bases, knowledge spaces, and knowledge graph.

(1) The architecture of Schema Centralized-Data Distributed allows numerous data to be stored as data silos in edge nodes to avoid frequent transmission, which gains the safety of critical data and reduces the bandwidth load of transmission.

(2) The mode of Dynamic-Knowledge solves the problem that knowledge is challenging to represent and reuse. IDMW combines domain knowledge and schema to subvert the traditional knowledge representing ways, which can be further used for the knowledge automation in advanced machining.

Additionally, there are some apparent differences between industrial dataspace and knowledge engineering, such as the knowledge graph. IDMW is defined in the context of data engineering, which mainly focuses on discovering the hidden relations of different data silos. While the knowledge graph of knowledge engineering, usually pays more intentions to represent explicit knowledge, which is intended for complex reasoning mainly.

\section{Prospects of the IDMW}

It is believed that the IDMW can achieve more profound and broader applications in the future. On the one hand, the latest graph technologies can be employed to discover more implied relations of the different data silos. For example, it is possible to reveal the relations of different workpieces with the same features but different machining techniques. On the other hand, an intelligent question-answering system can also be implemented in the Application Layer, by developing the machine-readable and machine-operable APPs, thereby realizing the Industry 4.0 oriented IDMW.

\section{Conclusions}

This paper presents an innovative conception of industrial dataspace together with its implementation framework against the workshop-level MOC. The architecture of the IDMW and its construction process have been discussed in detail.

The novel IDMW contributes to dealing with the challenging issues, particularly in managing heterogeneous data, interconnecting the resource entities, and handling domain knowledge within a machining workshop. Besides, this study shows how to construct the Process-Workpiece-Centric knowledge schema in IDMW and illustrates how it works by introducing framework construction, relation modeling, data storage, timelines determination, data silos pre-processing and domain knowledge reuse. Furthermore, through two industrial case studies, it is found that IDMW has effects on managing heterogeneous data, interconnecting resource entities and handling in-process knowledge.

Knowledge contributions of this research work lie in three aspects. The first is proposing modeling architecture of SchemaCentralized-DataDistributed. The second is discovering relations of data silos with the help of the RFID event graphical deduction model. The last is realizing the universal storage and processing domain knowledge in machining, even in the associated manufacturing process chain. 
Acknowledgements The research work is under the financial supports of the Natural Science Foundation of China with Grant Nos. 51975464 and 71571142. Thanks are also extended to the China Scholarships Council (CSC) and Brunel University London for hosting the academic scholarship.

\section{Compliance with ethical standards}

Conflict of interest The authors declare that they have no conflict of interest.

\section{References}

Ahmadian, A. S., Jürjens, J., \& Strüber, D. (2018). Extending modelbased privacy analysis for the industrial data space by exploiting privacy level agreements. In Paper presented at the proceedings of the 33rd annual ACM symposium on applied computing, Pau, France.

Angrish, A., Starly, B., Lee, Y., \& Cohen, P. H. (2017). A flexible data schema and system architecture for the virtualization of manufacturing machines (VMM). Journal of Manufacturing Systems, 45, 236-247. https://doi.org/10.1016/j.jmsy.2017.10.003.

Babiceanu, R. F., \& Seker, R. (2016). Big Data and virtualization for manufacturing cyber-physical systems: A survey of the current status and future outlook. Computers in Industry, 81, 128-137. https://doi.org/10.1016/j.compind.2016.02.004.

Belhajjame, K., Paton, N. W., Embury, S. M., Fernandes, A. A. A., \& Hedeler, C. (2013). Incrementally improving dataspaces based on user feedback. Information Systems, 38(5), 656-687. https://doi. org/10.1016/j.is.2013.01.006.

Chhim, P., Chinnam, R. B., \& Sadawi, N. (2019). Product design and manufacturing process based ontology for manufacturing knowledge reuse. Journal of Intelligent Manufacturing, 30(2), 905-916. https://doi.org/10.1007/s10845-016-1290-2.

Cui, Y., Kara, S., \& Chan, K. C. (2020). Manufacturing big data ecosystem: A systematic literature review. Robotics and ComputerIntegrated Manufacturing, 62, 101861. https://doi.org/10.1016/ j.rcim.2019.101861.

Curry, E. (2020). Real-time linked dataspaces-Enabling data ecosystems for intelligent systems. Cham: Springer.

Evans, R. D., Gao, J. X., Martin, N., \& Simmonds, C. (2017). A new paradigm for virtual knowledge sharing in product development based on emergent social software platforms. Proceedings of the Institution of Mechanical Engineers, Part B: Journal of Engineering Manufacture, 232(13), 2297-2308. https://doi.org/10.1177/0 954405417699018.

Flyverbom, M., Deibert, R., \& Matten, D. (2017). The governance of digital technology, big data, and the internet: New roles and responsibilities for business. Business and Society, 58(1), 3-19. https://doi.org/10.1177/0007650317727540.

Franklin, M., Halevy, A., \& Maier, D. (2005). From databases to dataspaces: A new abstraction for information management. Sigmod Record, 34(4), 27-33. https://doi.org/10.1145/1107499.1107502.

Gao, J., \& Nee, A. Y. (2017). An overview of manufacturing knowledge sharing in the product development process. Proceedings of the Institution of Mechanical Engineers, Part B: Journal of Engineering Manufacture, 232(13), 2253-2263. https://doi.org/10.11 $77 / 0954405418759375$.

He, L., \& Jiang, P. (2019). Manufacturing knowledge graph: A connectivism to answer production problems query with knowledge reuse. IEEE Access, 7, 101231-101244. https://doi.org/10.1109/ ACCESS.2019.2931361.
Ji, W., Yin, S., \& Wang, L. (2019). A big data analytics based machining optimisation approach. Journal of Intelligent Manufacturing, 30(3), 1483-1495. https://doi.org/10.1007/s10845-018-1440-9.

Jiang, P., Liu, C., Li, P., \& Shi, H. (2019). Industrial dataspace: A broker to run cyber-physical-social production system in level of machining workshops. In Paper presented at the 2019 IEEE 15th international conference on automation science and engineering (CASE), Vancouver, BC, Canada, 2019-01-01.

Katchasuwanmanee, K., Bateman, R., \& Cheng, K. (2015). Development of the energy-smart production management system (e-ProMan): A big data driven approach, analysis and optimisation. Proceedings of the Institution of Mechanical Engineers, Part B: Journal of Engineering Manufacture, 230(5), 972-978. https://doi.org/10.1177/0954405415586711.

Kovalenko, I., Tilbury, D., \& Barton, K. (2019). The model-based product agent: A control oriented architecture for intelligent products in multi-agent manufacturing systems. Control Engineering Practice, 86, 105-117. https://doi.org/10.1016/j.conengprac.2019.03. 009.

Lee, J., Bagheri, B., \& Kao, H. (2015). A cyber-physical systems architecture for industry 4.0-based manufacturing systems. Manufacturing Letters, 3, 18-23. https://doi.org/10.1016/j.mfglet.201 4.12.001.

Leng, J., Zhang, H., Yan, D., Liu, Q., Chen, X., \& Zhang, D. (2019). Digital twin-driven manufacturing cyber-physical system for parallel controlling of smart workshop. Journal of Ambient Intelligence and Humanized Computing, 10(3SI), 1155-1166. https://doi.org/10.1007/s12652-018-0881-5.

Li, P., \& Jiang, P. (2017). Knowledge-based innovative methods for collaborative quality control in equipment outsourcing chain. In Paper presented at the 2017 12th international conference on intelligent systems and knowledge engineering (ISKE), Nanjing, China, 2017-01-01.

Li, P., \& Jiang, P. (2019). Sensitivity analysis-based process stability evaluation for one-of-a-kind production. Proceedings of the Institution of Mechanical Engineers, Part C: Journal of Mechanical Engineering Science, 233(1), 63-77. https://doi.org/10.1177/095 4406218756939.

Li, P., Jiang, P., \& Liu, J. (2019). Mini-MES: A microservices-based apps system for data interconnecting and production controlling in decentralized manufacturing. Applied Sciences, 9(18), 3675. https://doi.org/10.3390/app9183675.

Liu, C., Jiang, P., \& Jiang, W. (2020). Web-based digital twin modeling and remote control of cyber-physical production systems. Robotics and Computer-Integrated Manufacturing, 64, 101956. https://doi.org/10.1016/j.rcim.2020.101956.

Liu, Y., Peng, Y., Wang, B., Yao, S., \& Liu, Z. (2017). Review on cyberphysical systems. IEEE/CAA Journal of Automatica Sinica, 4(1), 27-40. https://doi.org/10.1109/JAS.2017.7510349.

Lu, Y., Liu, C., Wang, K. I., Huang, H., \& Xu, X. (2020). Digital Twin-driven smart manufacturing: Connotation, reference model, applications and research issues. Robotics and ComputerIntegrated Manufacturing, 61, 101837. https://doi.org/10.1016/j. rcim.2019.101837.

Lu, Y., Wang, H., \& Xu, X. (2019). ManuService ontology: A product data model for service-oriented business interactions in a cloud manufacturing environment. Journal of Intelligent Manufacturing, 30(1), 317-334. https://doi.org/10.1007/s10845-016-1250-x.

Lu, Y., \& Xu, X. (2018). Resource virtualization: A core technology for developing cyber-physical production systems. Journal of Manufacturing Systems, 47, 128-140. https://doi.org/10.1016/j.jmsy.2 018.05.003.

McHugh, J., Cuddihy, P. E., Williams, J. W., Aggour, K. S., Kumar, V. S., \& Mulwad, V. (2017). Integrated access to big data polystores through a knowledge-driven framework. In Paper presented at the 
IEEE 2017 IEEE international conference on big data (Big Data), Boston, MA, USA, 2017-01-01.

Mezgebe, T. T., Demesure, G., Bril El Haouzi, H., Pannequin, R., \& Thomas, A. (2019). CoMM: A consensus algorithm for multiagent-based manufacturing system to deal with perturbation. The International Journal of Advanced Manufacturing Technology. https://doi.org/10.1007/s00170-019-03820-0.

Mirza, H. T., Chen, L., \& Chen, G. (2010). Practicability of dataspace systems. International Journal of Digital Content Technology and its Applications, 4(3), 233-243. https://doi.org/10.4156/jdcta. vol4.issue3.23.

Monostori, L., Kádár, B., Bauernhansl, T., Kondoh, S., Kumara, S., Reinhart, G., et al. (2016). Cyber-physical systems in manufacturing. CIRP Annals, 65(2), 621-641. https://doi.org/10.1016/j. cirp.2016.06.005.

Nickel, M., Murphy, K., Tresp, V., \& Gabrilovich, E. (2016). A review of relational machine learning for knowledge graphs. Proceedings of the IEEE, 104(1), 11-33. https://doi.org/10.1109/JPROC.201 5.2483592.

Niinimaki, M., \& Thanisch, P. (2019). Dataspace management for large data sets. In Innovative computing trends and applications (pp. 13-21). Springer, Cham.

Novas, J. M., Bahtiar, R., Van Belle, J., \& Valckenaers, P. (2012). An approach for the integration of a scheduling system and a multiagent manufacturing execution system. Towards a collaborative framework. IFAC Proceedings Volumes, 45(6), 728-733. https:// doi.org/10.3182/20120523-3-ro-2023.00156.

Parthiban, K., \& Nataraj, R. V. (2019). An efficient architecture to ensure data integrity in ERP systems. In Paper presented at the 20195 th international conference on advanced computing \& communication systems (ICACCS), Tamil Nadu, India, 2019-01-01.

Pullmann, J., Petersen, N., Mader, C., Lohmann, S., \& Kemeny, Z. (2017). Ontology-based information modelling in the industrial data space. In Paper presented at the 2017 22nd IEEE international conference on emerging technologies and factory automation (ETFA), Limassol, Cyprus, 2017-01-01.

Qi, Q., \& Tao, F. (2018). Digital twin and big data towards smart manufacturing and industry 4.0: 360 degree comparison. IEEE Access, 6, 3585-3593. https://doi.org/10.1109/ACCESS.2018.2793265.

Qiu, R. G., \& Zhou, M. (2004). Mighty MESs; state-of-the-art and future manufacturing execution systems. IEEE Robotics and Automation Magazine, 11(1), 19-25. https://doi.org/10.110 9/MRA.2004.1275947.

Song, Q., Wu, Y., Lin, P., Dong, L. X., \& Sun, H. (2018). Mining summaries for knowledge graph search. IEEE Transactions on Knowledge and Data Engineering, 30(10), 1887-1900. https:// doi.org/10.1109/TKDE.2018.2807442.

Tao, F., Qi, Q., Liu, A., \& Kusiak, A. (2018). Data-driven smart manufacturing. Journal of Manufacturing Systems, 48, 157-169. https://doi.org/10.1016/j.jmsy.2018.01.006.

UK, T. S. B. (2012). A landscape for the future of high value manufacturing in the UK. UK Technology Strategy Board Report.

Vogt, L., Baum, R., Köhler, C., Meid, S., Quast, B., \& Grobe, P. (2019). Using semantic programming for developing a web content management system for semantic phenotype data. In Paper presented at the international conference on data integration in the life sciences 2018, Hannover, Germany, 2019-01-01.
Wang, Y., Blache, R., Zheng, P., \& Xu, X. (2018a). A knowledge management system to support design for additive manufacturing using bayesian networks. Journal of Mechanical Design. https:// doi.org/10.1115/1.4039201.

Wang, C., Jiang, P., \& Lu, T. (2018b). Production events graphical deduction model enabled real-time production control system for smart job shop. Proceedings of the Institution of Mechanical Engineers, Part C: Journal of Mechanical Engineering Science, 232(16), 2803-2820. https://doi.org/10.1177/095440621772853 1.

Wang, S., Wan, J., Zhang, D., Li, D., \& Zhang, C. (2016). Towards smart factory for industry 4.0: A self-organized multi-agent system with big data based feedback and coordination. Computer Networks, 101, 158-168. https://doi.org/10.1016/j.comnet.2015.12.017.

Xiao, G., Calvanese, D., Kontchakov, R., Lembo, D., Poggi, A., \& Rosati, R., et al. (2018). Ontology-based data access: A survey. In Paper presented at the proceedings of the 27th international joint conference on artificial intelligence, Stockholm, Sweden.

Xu, L. D., \& Duan, L. (2019). Big data for cyber physical systems in industry 4.0: A survey. Enterprise Information Systems, 13(2), 148-169. https://doi.org/10.1080/17517575.2018.1442934.

Ye, F., \& Wang, Z. (2013). Effects of information technology alignment and information sharing on supply chain operational performance. Computers \& Industrial Engineering, 65(3), 370-377. https://doi. org/10.1016/j.cie.2013.03.012.

Zammit, J., Gao, J., Evans, R., \& Maropoulos, P. (2017). A knowledge capturing and sharing framework for improving the testing processes in global product development using storytelling and video sharing. Proceedings of the Institution of Mechanical Engineers, Part B: Journal of Engineering Manufacture, 232(13), 2286-2296. https://doi.org/10.1177/0954405417694062.

Zhang, C., Jiang, P., Cheng, K., Xu, X. W., \& Ma, Y. (2016). Configuration design of the add-on cyber-physical system with CNC machine tools and its application perspectives. Procedia CIRP, 56, 360-365. https://doi.org/10.1016/j.procir.2016.10.040.

Zhong, R. Y., Xu, C., Chen, C., \& Huang, G. Q. (2017a). Big data analytics for physical internet-based intelligent manufacturing shop floors. International Journal of Production Research, 55(9), 2610-2621. https://doi.org/10.1080/00207543.2015.1086037.

Zhong, R. Y., Xu, X., Klotz, E., \& Newman, S. T. (2017b). Intelligent manufacturing in the context of industry 4.0: A review. Engineering, 3(5), 616-630. https://doi.org/10.1016/j.eng.2017.05.015.

Zhou, J., Li, P., Zhou, Y., Wang, B., Zang, J., \& Meng, L. (2018). Toward new-generation intelligent manufacturing. Engineering, 4(1), 11-20. https://doi.org/10.1016/j.eng.2018.01.002.

Publisher's Note Springer Nature remains neutral with regard to jurisdictional claims in published maps and institutional affiliations. 